$05,12,13$

\title{
Управляемая электрическим полем спин-волновая связь в латеральных ансамблях магнитных микроструктур
}

\author{
(C) А.А. Грачев, Е.Н. Бегинин, С.Е. Шешукова, А.В. Садовников \\ Саратовский государственный университет им. Н.Г. Чернышевского, \\ Саратов, Россия \\ E-mail: stig133@gmail.com
}

Поступила в Редакцию 9 апреля 2021 г.

В окончательной редакции 9 апреля 2021 г.

Принята к публикации 19 апреля 2021 г.

\begin{abstract}
С помощью численных и экспериментальных исследований продемонстрирована возможность управления дипольной спин-волновой связью в латеральном массиве ферритовых полосок с помощью локальных деформаций. В качестве экспериментальной демонстрации исследованных физических процессов предложена конфигурация магнонной структуры с пьезоэлектрическим слоем и структурированными электродами на его поверхности и использована методика лазерной абляции с пространственным разрешением для структурирования пьезоэлектрического слоя. Показано, что с помощью локальных упругих деформаций оказывается возможным индуцировать волноведущий канал для распространяющихся спин-волновых краевых мод. С прикладной точки зрения, полученные результаты могут быть использованы для создания класса устройств обработки информации, таких как системы демультиплексирования с частотно-пространственной селективностью, управляемых одновременно электрическим и магнитным полем.
\end{abstract}

Ключевые слова: спиновые волны, магноника, стрейнтроника, латеральные структуры.

DOI: $10.21883 /$ FTT.2021.09.51253.14H

\section{1. Введение}

В настоящее время использование заряда носителей тока (электронов или дырок) является основным эффектом в полупроводниковой микроэлектронике. Использование элементарных квантов магнитных возбуждений (магнонов) и спиновых волн (СВ) в диэлектрических магнитных пленках, поддерживают передачу сигнала без движения зарядов и, следовательно, без омических потерь, обеспечивают сверхнизкое энергопотребление и являются многообещающей альтернативой полупроводниковым приборам [1-3]. Одним из новых научных направлений в физике конденсированного состояния является магноника [4-6], ставящая перед собой задачи по исследованию методов и характеристик управления спиновыми волнами в волноведущих системах в микрои наномасштабах $[7,8]$.

Недавние теоретические и экспериментальные исследования показывают, что деформация может быть использована для создания энергоэффективных сложных двухмерных и трехмерных пьезоэлектрических материалов и гетероструктур на основе полупроводников [9], сегнетоэлектриков [10], графеновых слоев [11] и массивов нанопроволок [13]. Было также показано, что можно индуцировать сдвиг частоты ферромагнитного резонанса из-за влияния преобразования электрического поля в магнитное поле [13]. Влияние электрического поля на магнитную конфигурацию обусловлено модификацией эффективного внутреннего магнитного поля. Последнее изменяется из-за обратной магнитострикции (эффект Виллари) в результате локальной деформации магнит- ной пленки. Экспериментально показано, что спинволновая связь может эффективно использоваться для управления переносом магнонов [14], что привело к созданию класса спин-волновых устройств, таких как, двухканальные направленные ответвители [15], спинволновые сплиттеры [16,17].

В настоящей работе с помощью численных и экспериментальных исследований продемонстрированы эффекты управления спин-волновой связью в системе из трех латеральных магнитных полосок с пьезоэлектрическим слоем. Выявлены механизмы управления дипольной связью спиновых волн путем создания упругих деформаций, локализованных в области максимумов напряженности электрического поля. Показана эффективная перестройка спин-волновых характеристик с помощью электрического поля, обусловленная локальной деформацией пьезослоя и обратным эффектом магнитострикции в ферритовых микрополосках и выявлены механизмы управления электрическим полем режимами пространственночастотной селекции СВ.

\section{2. Структура и численное исследование}

Для экспериментального исследования механизмов управления спин-волновой связью была изготовлена латеральная структура, представляющая собой три параллельно ориентированных магнитных полоски $Z_{1}, Z_{2}$ и $Z_{3}$, образующих три спин-волновых канала (рис. 1). C помощью метода лазерного скрайбирования из пленки 


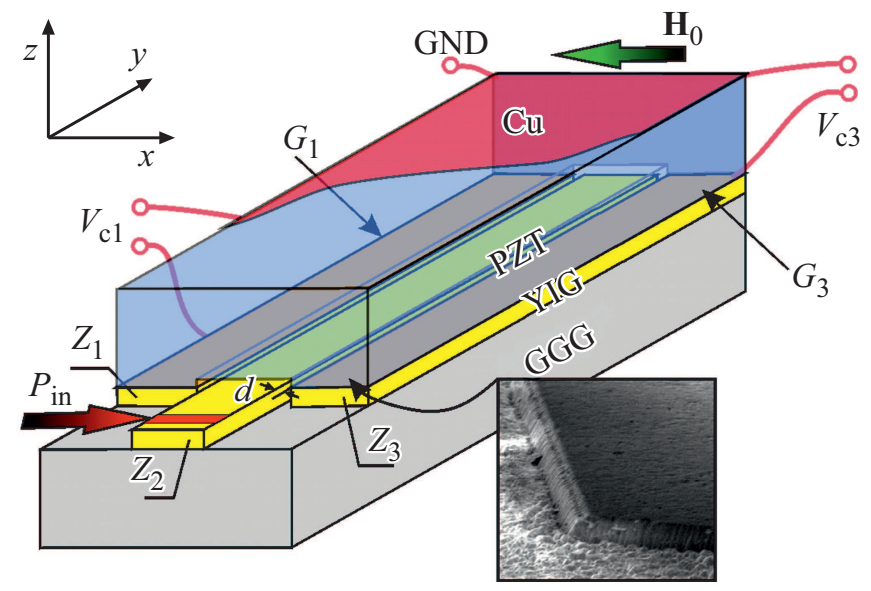

Рис. 1. Схема рассматриваемой структуры. На вставке приведено SEM-изображение края пьезоэлектрического слоя.

железо-иттриевого граната (ЖИГ) толщиной $t=10 \mu \mathrm{m}$ $\left[(\mathrm{YIG}) \mathrm{Y}_{3} \mathrm{Fe}_{5} \mathrm{O}_{12}(111)\right]$ на подложке из галлий-гадолиниевого граната (ГГГ) $\left[(\mathrm{GGG}) \mathrm{Gd}_{3} \mathrm{Ga}_{5} \mathrm{O}_{12}\right.$ (111) толщиной $500 \mu \mathrm{m}$ сформирована система латеральных микроволноводов шириной $w=500 \mu \mathrm{m}$ с расстоянием $d=40 \mu \mathrm{m}$ между ними. Длина вдоль длинной стороны волноводов составляла $6 \mathrm{~mm}$ для $Z_{1,3}$ и $8 \mathrm{~mm}$ для $Z_{2}$. Возбуждение СВ осуществлялось с помощью микрополосковой антенны толщиной $1 \mu \mathrm{m}$ и шириной $30 \mu \mathrm{m}$. Структура помещена во внешнее статическое магнитное поле, $H_{0}=1100$ Ое, ориентированное вдоль оси $x$ для эффективного возбуждения поверхностной магнитостатической волны (ПМСВ) в $Z_{2}$. На верхней поверхности пьезоэлектрического керамического слоя из цирконататитаната свинца (ЦТС) $\left(\left(\mathrm{Pb}\left(\mathrm{Zr}_{0.3} \mathrm{Ti}_{0.7}\right) \mathrm{O}_{3}\right)\right.$ PZT) с размерами $1580 \times 4000 \times 200 \mu \mathrm{m}^{3}$ размещен электрод (обозначение „GND“ на рис. $1, a)$ из меди толщиной $1 \mu \mathrm{m}$, который не оказывает значительного влияния на распространение СВ в магнитных полосках. На другой стороне ЦТС были напылены электроды $G_{1}$ и $G_{3}$ из титана, толщиной $100 \mathrm{~nm}$, расположенные над $Z_{1}$ и $Z_{3}$ соответственно. Отдельно к каждому из электродов в эксперименте прикладывалось напряжение величиной $V_{c 1,3}$. Для более эффективного управления спин-волновым транспортом с помощью локальных деформаций, впервые использована методика лазерной абляции с пространственным разрешением для вытравливания пьезоэлектрического слоя толщиной $25 \mu \mathrm{m}$ и формирования трапецеидальных электродов для улучшения пьезомагнитной связи, в виду локального уменьшения толщины слоя ЦТС. На вставке на рис. $1, a$ показано изображение с электронного микроскопа, демонстрирующее края пьезоэлектрического слоя, находящегося в непосредственном контакте с ЖИГ полоской. В эксперименте эффективная механическая связь между полосками ЖИГ и ЦТС слоем была достигнута с помощью использования двухкомпонентного эпоксидного тензометрического клея.
Для описания физических процессов, определяющих физические характеристики при управлении спин-волновыми сигналами путем создания локальных упругих деформаций, была разработана численная модель на основе метода конечных элементов (МКЭ). На первом этапе выполнялся расчет упругих деформаций, вызываемых внешним электрическим полем в слое пьезоэлектрика. Далее, рассчитывались профили внутреннего магнитного поля в латеральных магнитных полосках. Затем, полученные профили внутреннего магнитного поля использовались в микромагнитном моделировании [18] и расчете спектров собственных мод поперечноограниченных магнитных полосок с помощью МКЭ [19]. Относительное изменение размеров слоя ЦТС показано на рис. $2, a$, где градациями цвета изображено распределение значений компоненты тензора механических напряжений $S_{\mathrm{xx}}$ в случае $V_{c 1,3}= \pm 250 \mathrm{~V}$. Можно отметить, что деформация пьезоэлектрического слоя возникает в локальной области слоя ЦТС под электродами $G_{1,3}$, что приводит к изменению величины внутреннего магнитного поля $H_{\text {int }}$ в полосках $Z_{1,3}$ вследствие обратного магнитострикционного эффекта. Была проведена оценка влияния деформаций структурированного (левая панель на рис. $2, a$ ) и не структурированного пьезослоя (правая панель на рис. $2, a$ ). Видно, что в случае структурированного слоя пьезоэлектрика наблюдается усиление локальных деформаций в области контакта пьезоэлектрического слоя с ЖИГ полоской.
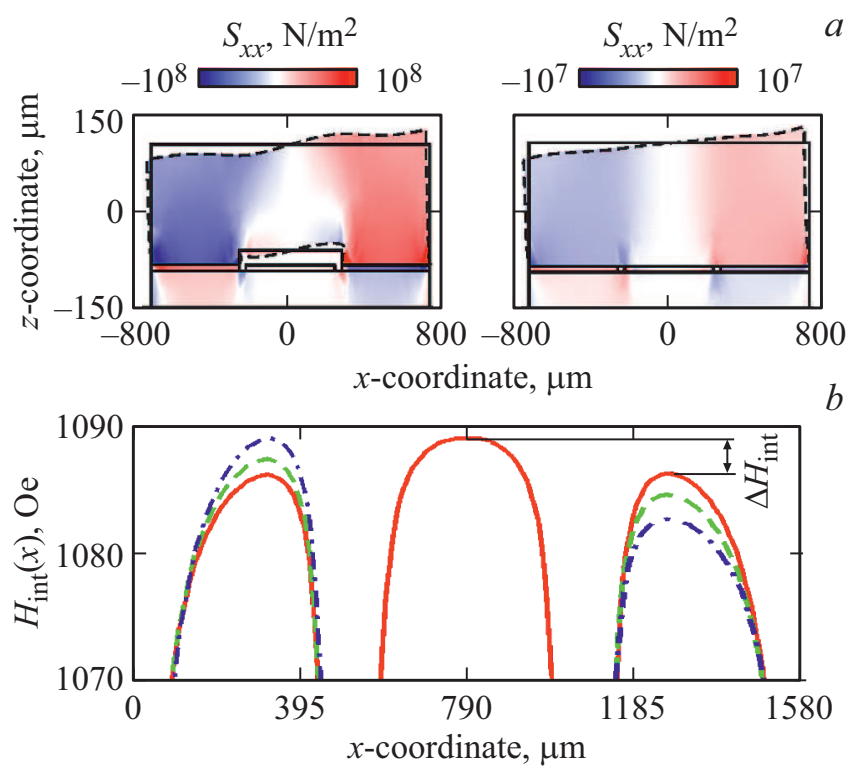

Рис. 2. $a-$ распределения компоненты тензора механических напряжений $S_{\mathrm{xx}}$ при приложении к электродам $G_{1,3}$ напряжения $V_{c 1,3}= \pm 250 \mathrm{~V}$ в случае структурированного (левая панель) и не структурированного (правая панель) пьезоэлектрического слоя. $b-$ профили внутреннего магнитного поля $H_{\text {int }}$ в случаях $E_{1,3}=0 \mathrm{kV} / \mathrm{cm}$ (сплошные кривые); для не структурированного пьезослоя при $E_{1}=-10 \mathrm{kV} / \mathrm{cm}, E_{3}=10 \mathrm{kV} / \mathrm{cm}$; для структурированного пьезослоя при $E_{1}=-10 \mathrm{kV} / \mathrm{cm}, E_{3}=10 \mathrm{kV} / \mathrm{cm}$. Величина внешнего магнитного поля $H_{0}=1100 \mathrm{Oe}$. 

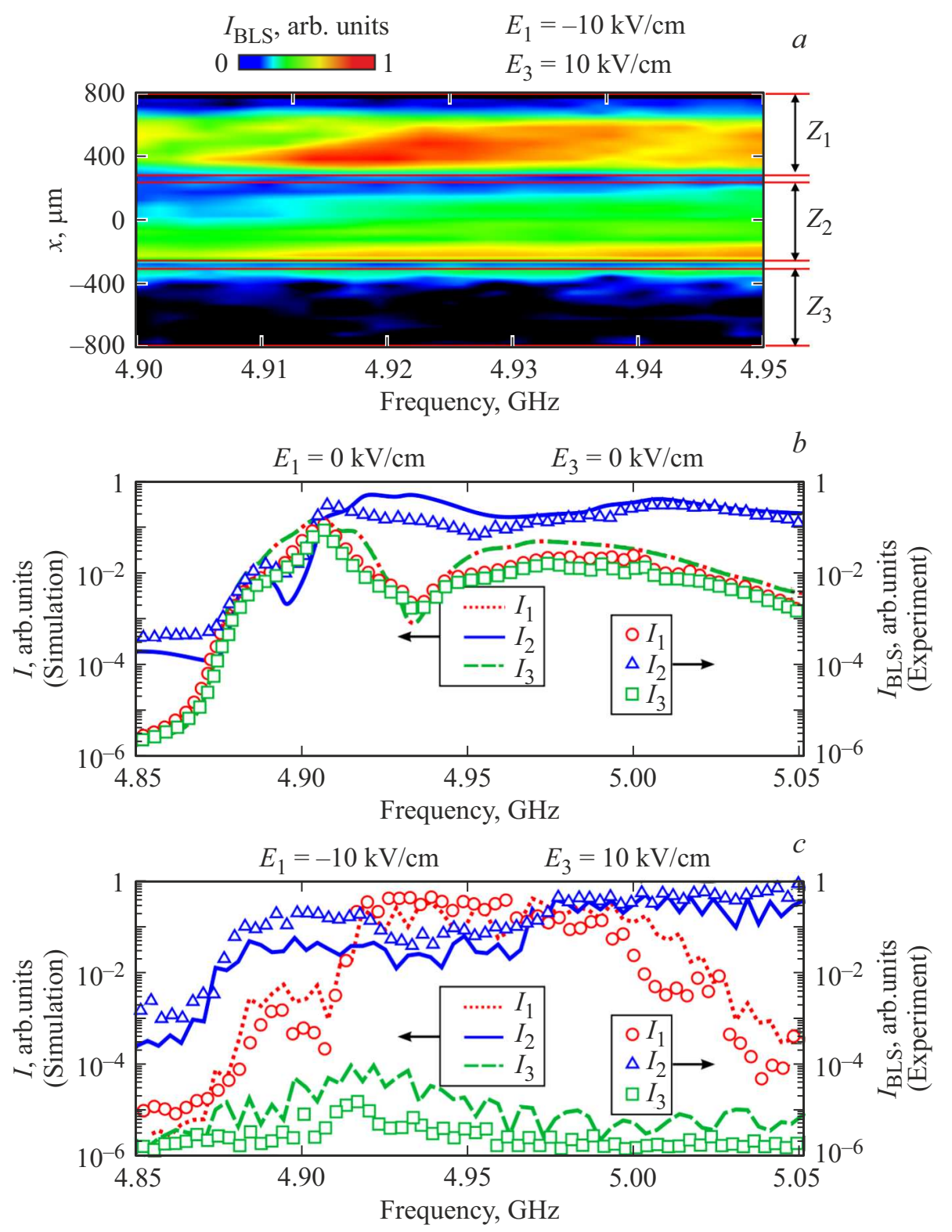

Рис. 3. Частотная зависимость МБС-сигнала $(a)$ в сечении $y=3 \mathrm{~mm}$ при $E_{1}=-10 \mathrm{kV} / \mathrm{cm}$ и $E_{3}=10 \mathrm{kV} / \mathrm{cm}$. Частотный спектр спин-волнового сигнала в выходной секции магнитных полосок $Z_{1,2,3}$ при $E_{1}=E_{3}=0(b), E_{1}=-10 \mathrm{kV} / \mathrm{cm} \mathrm{и} E_{3}=10 \mathrm{kV} / \mathrm{cm}(c)-$ результат МБС (символы, правая вертикальная ось) и микромагнитных вычислений (кривые, левая вертикальная ось).

Стоит отметить, что эффективное управление свойствами $\mathrm{CB}$, распространяющихся в латеральной структуре, с помощью локальных упругих деформаций оказывается возможным благодаря неоднородному распределению величины $H_{\text {int. }}$ Когда магнитное поле $H_{0}$ направлено вдоль оси $x$, распределение $H_{\text {int }}(x)$ в латеральных магнитных полосках становится неоднородным из-за анизотропии формы, что приводит к формированию трех спин-волновых каналов, вдоль которых распространяются СВ (см. рис. 2,b). Приложение положительного электрического поля к электроду $G_{3}$ и отрицательного поля к $G_{1}$ приводит к уменьшению внутреннего магнитного поля в $Z_{1}$ и увеличению Hint в полоске $Z_{3}$, как показано на рис. $2, b$. Статическое распределение магнитного поля в структуре определяется величиной намагниченности ЖИГ и геометрическими размерами магнитных полосок, при этом малое изменение величины расстояния между латеральными полосками $d$ не приводит к значительному изменению величины $\Delta H_{\text {int }}$, однако приводит к изменению величины связи между $\mathrm{CB}$, распространяющимися в $Z_{1,2,3}$. Следует отметить, что в случае структурированного слоя пьезоэлеткрика (см. штрих-пунктирные кривые на рис. $2, b$ ) наблюдается более эффективное изменение внутреннего магнитного поля. Действительно влияния эффекта Виллари проявляется в изменении внутреннего поля в одном из микроволноводов, но это приводит к тому, что меняется профиль собственной моды в этом волноводе, будь 
он изолированным от других рядом расположенных. Это означает, что изменится профиль „провисающих“ за границы микроволновода электромагнитных полей. Последнее в свою очередь приведет к тому, что поднесенный к такому волноводу соседний микроволновод будет испытывать влияния этого „провисающего поля“ несколько иначе, чем если бы он был расположен рядом с полностью идентичным ему микроволноводом. В этом смысле, можно указать на изменение величины дипольной связи, влияющей на результирующую картину, возникающую при связи спиновых волн и их перераспределении мощности, при распространении вдоль микроволноводов. Другими словами, эффект Виллари приводит к изменению профилей собственных мод и дисперсионных характеристик, соответствующих этим модам, что можно трактовать как изменение связи между микроволноводами. На языке теории связанных волн это можно записать, как зависимость коэффициента связи между волнами в двух (трех и т.д.) микроволноводах от величины внутреннего поля в одном из них.

\section{3. Экспериментальное исследование}

Экспериментальное исследование влияния локальных упругих деформаций на стационарное распределение интенсивности ПМСВ проводилось методом мандельштамбриллюэновской спектроскопии (МБC, BLS) магнитных материалов [20]. Зондирующий лазерный луч с длиной волны $532 \mathrm{~nm}$ был сфокусирован на прозрачной стороне ГГГ композитной структуры. Чтобы показать трансформацию поперечного распределения интенсивности СВ при приложении электрического поля, была получена частотная зависимость величины $I_{\mathrm{BLS}} \sim \sqrt{\beta_{1} m_{y}^{2}+\beta_{2} m_{z}^{2}}$ (константы $\beta_{1}$ и $\beta_{2}$ определяются геометрией рассеяния) в сечении вдоль оси $x$ при $y=3.0 \mathrm{~mm}$, в случае $E_{1}=-10 \mathrm{kV} / \mathrm{cm}$ и $E_{3}=10 \mathrm{kV} / \mathrm{cm}$ (см. рис. $\left.3, a\right)$. Видно, что в данном случае наблюдается перераспределение мощности $\mathrm{CB}$ в полосках $Z_{2,3}$. При этом в полоске $Z_{2}$ распространяется краевая мода и наблюдается связь между краевыми модами, распространяющимися вдоль $Z_{2}$ и $Z_{3}$. Следует отметить, что краевая мода возбуждается в полоске $Z_{3}$ в диапазоне частот 4.9-4.95 GHz, как показано на рис. 3, a. Поэтому распространение центральной моды вдоль $Z_{3}$ подавляется локально созданной деформацией. Таким образом с помощью локальных упругих деформаций оказывается возможным индуцировать волноведущий канал для распространяющихся спин-волновых краевых мод.

На рис. 3 построены частотные зависимости интенсивности динамической намагниченности $I_{j}$ при $y=3.0 \mathrm{~mm}$ на выходе каждого из волноводов $Z_{j}(j=1,2,3)$, полученные методом микромагнитного моделирования (линии) и в эксперименте (точки) для случая $E_{1}=E_{3}=0$ (см. рис. $3, b)$, а также зависимости для $E_{1}=-10 \mathrm{kV} / \mathrm{cm}$ и $E_{3}=10 \mathrm{kV} / \mathrm{cm}$ (см. рис. $3, c$ ) для качественного и количественного сравнения. Видно качественное соответствие данных, полученный в эксперименте и при численном моделировании, при этом в случае $E_{1}=-10 \mathrm{kV} / \mathrm{cm}$ и $E_{3}=10 \mathrm{kV} / \mathrm{cm}$ мощность СВ локализована в полосках $Z_{1}$ и $Z_{2}$. Разница в данных МБС эксперимента и численного моделирования может быть объяснена учетом конечного числа поперечных (ширинных) мод в численном счете. При этом, МБС сигнал является результатом интерференции как четных, так и нечетных ширинных мод отдельно взятой магнитной полоски, поскольку рассматриваемая структура не является трансляционно инвариантной вдоль оси $y$. Видно, что в случае отсутствия внешнего электрического поля в системе наблюдается симметричный перенос спинволновой энергии из полоски $Z_{2}$ в $Z_{1,3}$. При приложении электрического поля к слою пьезоэлектрика происходит трансформация передаточных характеристик происходит нарушение симметрии характеристик для $Z_{1,3}$. Следует отметить, что в данном случае наблюдается потеря мощности $\mathrm{CB}$ в полоске $Z_{3}$ и мощность спиновой волны перераспределяется между полосками $Z_{1,2}$.

\section{4. Заключение}

Таким образом, управляемый электрическим напряжением спин-волновой транспорт может быть реализован в структурированных многослойных пленках ферритпьезоэлектрик. При этом формирование электродов на поверхности пьезослоя позволяет создавать упругие деформации в области неоднородности внутреннего магнитного поля в пленке феррита. Последняя при этом создается путем структурирования поверхности магнитной пленки и создания на ней нерегулярных спин-волноведущих каналов. В качестве демонстрации данного физического эффекта, используя численные и экспериментальные методы, показан управляемый напряжением спинволновой транспорт вдоль трехканальной латеральной структуры. Показана эффективная перестройка спинволновых характеристик с помощью электрического поля, обусловленным деформацией пьезослоя и эффектом магнитострикции в ЖИГ-полосках. Показано, что с помощью локальных упругих деформаций оказывается возможным индуцировать волноведущий канал для распространяющихся спин-волновых краевых мод. Рассмотренный в работе эффект может быть положен в основу конструктивного решения для создания, управляемого демультиплексера, когда для различных частот входного сигнала выходной сигнал будет в разных портах многоканальной структуры при возможности перестройки величин мощностей между выходными портами (путем приложения электрического поля).

\section{Финансирование работы}

Работа выполнена при поддержке Минобрнауки России в рамках выполнения государственного задания (проект № FSRR-2020-0005).

\section{Конфликт интересов}

Авторы заявляют, что у них нет конфликта интересов. 


\section{Список литературы}

[1] S. Neusser, D. Grundler. Adv. Mater. 21, 2927 (2009).

[2] S.A. Nikitov, A.R. Safin, D.V. Kalyabin, A.V. Sadovnikov, E.N. Beginin, M.V. Logunov, M.A. Morozova, S.A. Odintsov, S.A. Osokin, A.Yu. Sharaevskaya, Yu.P. Sharaevsky, A.I. Kirilyuk. Phys. Usp. 63, 945 (2020).

[3] D. Sander, S.O. Valenzuela, D. Makarov, C.H. Marrows, E.E. Fullerton, P. Fischer, J. McCord, P. Vavassori, S. Mangin, P. Pirro, B. Hillebrands, A.D. Kent, T. Jungwirth, O. Gutfleisch, C.G. Kim, A. Berger. J. Phys. D 50, 363001 (2017).

[4] K. Roy, S. Bandyopadhyay, J. Atulasimha. Appl. Phys. Lett. 99, 063108 (2011).

[5] Yi-Pu. Wang, J.W. Rao, Y. Yang, Peng-Chao Xu, Y.S. Gui, B.M. Yao, J.Q. You, C.-M. Hu. Phys. Rev. Lett. 123, 127202 (2019).

[6] A.V. Sadovnikov, E.N. Beginin, S.E. Sheshukova, Yu.P. Sharaevskii, A.I. Stognij, N.N. Novitski, V.K. Sakharov, Yu.V. Khivintsev, S.A. Nikitov. Phys. Rev. B 99, 054424 (2019).

[7] P. Che, K. Baumgaertl, A. Kúkol'ová, C. Dubs, D. Grundler. Nature Commun. 11, 1445 (2020).

[8] P. Frey, A.A. Nikitin, D.A. Bozhko, S.A. Bunyaev, G.N. Kakazei, A.B. Ustinov, B.A. Kalinikos, F. Ciubotaru, A.V. Chumak, Q. Wang, V.S. Tiberkevich, B. Hillebrands, A.A. Serga. Commun. Phys. 3, 17 (2020).

[9] A.A. Shevyrin, A.G. Pogosov, A.K. Bakarov, A.A. Shklyaev. Phys. Rev. Lett. 117, 017702 (2016).

[10] S. Liu, R.E. Cohen. Phys. Rev. Lett. 119, 207601 (2017).

[11] N. Levy, S.A. Burke, K.L. Meaker, M. Panlasigui, A. Zettl, F. Guinea, A.H.C. Neto, M.F. Crommie. Science 329, 544 (2010).

[12] Z.L. Wang, J. Song. Science 312, 242 (2006).

[13] Y.K. Fetisov, G. Srinivasan. Appl. Phys. Lett. 93, 033508 (2008).

[14] A.V. Sadovnikov, E.N. Beginin, S.E. Sheshukova, D.V. Romanenko, Y.P. Sharaevskii, S.A. Nikitov. Appl. Phys. Lett. 107, 202405 (2015).

[15] Q. Wang, P. Pirro, R. Verba, A. Slavin, B. Hillebrands, A.V. Chumak. Sci. Adv. 4, e1701517 (2018).

[16] A.V. Sadovnikov, A.A. Grachev, E.N. Beginin, S.E. Sheshukova, Y.P. Sharaevskii, S.A. Nikitov. Phys. Rev. Appl. 7, 014013 (2017).

[17] A.V. Sadovnikov, A.A. Grachev, S.E. Sheshukova, Y.P. Sharaevskii, A.A. Serdobintsev, D.M. Mitin, S.A. Nikitov. Phys. Rev. Lett. 120, 257203 (2018).

[18] A. Vansteenkiste, J. Leliaert, M. Dvornik, M. Helsen, F. Garcia-Sanchez, B. Van Waeyenberge. AIP Advances 4, 107133 (2014).

[19] P.P. Silvester, R.L. Ferrari. Finite Elements for Electrical Engineers, Cambridge University Press (1996), 541 p.

[20] S.O. Demokritov, B. Hillebrands, A.N. Slavin. Phys. Rep. 348, 441 (2001).

Редактор Т.Н. Василевская 